\title{
BROWNIAN MOTION, GEOMETRY, AND GENERALIZATIONS OF PICARD'S LITTLE THEOREM
}

\author{
BY S. I. GOLDBERG ${ }^{1}$ AND C. MUELLER ${ }^{2}$
}

\begin{abstract}
ABST RACT. Brownian motion is introduced as a tool in Riemannian geometry, and it is shown to be useful in the function theory of manifolds, as well as in the study of maps between manifolds. As applications, a generalization of Picard's little theorem, and a version of it for Riemann surfaces of large genus are given.
\end{abstract}

1. Picard's theorem for nonhyperbolic manifolds. Let $M$ and $N$ be complete Riemannian manifolds with metrics ${ }^{M_{g}},{ }^{N}$, resp. Assume $F: M \rightarrow N$ is a $C^{2}$ map. $F$ is said to be harmonic [2] if its second fundamental form has trace 0 . Define the tensor

$$
\xi^{\alpha \beta}(x)={ }^{M} g_{i j}\left[\frac{\partial F^{\alpha}}{\partial x^{i}} \frac{\partial F^{\beta}}{\partial x^{j}}\right](x), x \in M .
$$

Since $\left(\xi^{\alpha \beta}(x)\right)$ is a symmetric matrix, its eigenvalues are nonnegative, and we may order them as follows: $\lambda_{1}(x) \geqslant \lambda_{2}(x) \geqslant \cdots \geqslant \lambda_{n}(x) \geqslant 0$. $F$ is said to be $K$ quasiconformal [5] if $\lambda_{1}(x) \leqslant K^{2} \lambda_{n}(x)$ for all $x \in M$.

We define polar coordinates $(r, \theta)$ on $N$ via the exponential map. There will be two restrictions on the curvature of $N$ :

(i) The sectional curvatures of $N$ are bounded below by $-L^{2}<0$.

(ii) Each of the sectional curvatures at $(r, \theta) \in N$ determined by $d r$ and some other tangent vector, is bounded above by $K(r)$, where $K(r)$ satisfies (a) for some $\epsilon>0,-K(r) \sim r^{2 \epsilon-2}$; (b) there exists a $C^{\infty}$ solution $u(r)$ of the equation

$$
u^{\prime \prime}(r)=K(r) u(r), \quad u(0)=0, \quad u^{\prime}(0)=1,
$$

and $u^{\prime}(r)$ is always positive.

(Note that such a solution can always be found if $K(r)$ is negative.)

THEOREM 1. Suppose $M$ and $N$ are as above with the curvature of $N$ satisfying (i) and (ii). Then, if Brownian motion on $M$ has trivial tail $\sigma$-field, every $K$-quasiconformal harmonic map $F: M \longrightarrow N$ is constant.

Received by the editors January 26, 1982 and, in revised form, March 23, 1982.

1980 Mathematics Subject Classification. Primary 32H25, 53C21, $60 \mathrm{~J} 65$.

1 Queen's Quest Professor, Queen's University, Kingston, Canada.

2 NSF Postdoctoral Fellow, University of Illinois, Urbana, 1979-1981. 
2. Proof of Theorem 1. The following lemmas are essential.

Lemma 2. Let $X_{t}$ be Brownian motion on $M$, with $X_{0}$ chosen so that $F\left(X_{0}\right)=x_{N} \in M\left\{x_{0}\right\}$. Then, there is a random time change $\sigma(t)\left(=\int_{0}^{t} d s / \lambda_{1}\left(x_{s}\right)\right)$ and a constant $C>0$, such that if $\rho_{t}=r \circ F\left(X_{\sigma(t)}\right), d \rho_{t}=a\left(X_{\sigma(t)}\right) d B_{t}+b\left(X_{\sigma(t)}\right) d t$, when $F\left(X_{t}\right) \neq x_{0}$, where $B_{t}$ is some Brownian motion, $1 / K \leqslant\left|a\left(X_{\sigma(t)}\right)\right| \leqslant 1$ and $b\left(X_{\sigma(t)}\right) \geqslant C \rho_{t}^{\epsilon / 2-1}$ if $\rho_{t}$ is larger than some constant $R$.

The statement uses the stochastic calculus discussed in [8].

Let $\tau$ be a stopping time for $X_{\sigma(t)}$.

LEMMA 3. If $\rho_{t}^{(\tau)}$ is the distance of $F\left(X_{\sigma(t)}\right)$ from $F\left(X_{\sigma(\tau)}\right)$, then for $t>\tau$ and $\rho_{t}^{(\tau)} \neq 0, d \rho_{t}^{(\tau)}=a^{(\tau)}\left(X_{\sigma(t)}\right) d B_{t}^{(\tau)}+b^{(\tau)}\left(X_{\sigma(t)}\right) d t$, where $1 / K \leqslant\left|a^{(\tau)}\left(X_{\sigma(t)}\right)\right|$ $\leqslant 1$ and $\mid b^{(\tau)}\left(X_{\sigma(t)} \mid \leqslant(n / 2) L\right.$ coth $L \rho_{t}^{(\tau)}$.

The proofs of Lemmas 2 and 3 require Ito's lemma, the $K$-quasiconformal condition, and the Hessian comparison theorem of Greene and $\mathrm{Wu}$ [3]. Lemma 2 is used to determine the speed with which $\rho_{t}$ goes to $\infty$.

LEMMA 4. $\lim \inf _{t \rightarrow \infty} \rho_{t} / t^{2 / 4-\epsilon}>c>0$.

Lemma 4 will be required in the proof of

Lemma 5. Let $\tau_{Q}$ be the first time that $\rho_{t} \geqslant Q$. Then, for $h$ sufficiently large, there is a $q \in(0,1)$ and a constant $C_{1}$ such that

$$
P\left\{\rho_{t+\tau_{Q}}>C_{1}(t+h)^{2 / 4-\epsilon} \text { for } t>0 \mid X_{\sigma\left(\tau_{Q}\right)}\right\}>q
$$

uniformly in $X_{\sigma\left(\tau_{Q}\right)}$.

Lemma 6. Let $r=\inf (r(x), r(y))$ for $x, y \in N$. If $|\Theta(x, y)|$ denotes the angular distance between $\theta(x)$ and $\theta(y)$, then for some constants $C$ and $R, r>R$,

$$
|\Theta(x, y)| \leqslant C \frac{N_{d(x, y)}}{\exp \left(r^{\epsilon}\right)},
$$

where ${ }^{N} d$ denotes the distance in the manifold $N$.

Proof. We use the Rauch comparison theorem for the same comparison manifold $P$ as is used in the proof of Lemma 2 .

Leмma 7. There exists a random time $T$ such that for all $m>T$,

$$
\sup _{\sigma(m) \leqslant t \leqslant \sigma(m+1)} N d\left(F\left(X_{t}\right), F\left(X_{\sigma(m)}\right)\right) \leqslant m .
$$

Proof. Using Lemma 3, we must show, for $m>T$, that $\sup _{m \leqslant t \leqslant m+1} \rho_{t}^{(m)}$ $\leqslant m$. The argument is similar to an idea of Prat [11], and is also used by Kendall [7] and Pinsky [10]. 
LEMмA 8. Fix $\delta>0$. If $\tau$ is a stopping time, we may choose an integer $J$ so large that

$$
P\left\{\sup _{\tau \leqslant t-m \leqslant \tau+1} \rho_{t}^{(\tau+m)} \leqslant m+J, \text { for all } m \geqslant 1\right\} \geqslant 1-\delta .
$$

Lemmas 4-8, and a result of Stroock and Varadhan [12, Theorem 3.1] show that the tail $\sigma$-field $\lim _{t \rightarrow \infty} \theta\left(F\left(X_{t}\right)\right)$ of $X_{t}$ exists and is nontrivial. This is a contradiction, so $F$ must be constant.

3. Picard's theorem for Riemann surfaces of large genus. In the sequel, let $M$ and $N$ be compact Riemann surfaces with a finite number of points deleted. Any such surface $N$ is homeomorphic to a sphere with $t(N)$ tori attached and $p(N)$ points deleted. Let $n(N)=2 t(N)+p(N)$.

THEOREM 2. Let $F: M \rightarrow N$ be a holomorphic map. If $t(M)>0, p(M)$ $>0, p(N)>1$, and $n(N)-n(M)>0$, then $F$ is constant.

Proof of Theorem 2. The proof uses Brownian motion on Riemann surfaces, which can be defined as follows. Let $\left\{m_{i}\right\}$ be coordinate patches on $M$, and for each $i$, let $c_{i}: m_{i} \rightarrow \mathbf{C}$, where $\mathbf{C}$ is the complex plane, be a holomorphic map. For $p \in M$, choose a patch $m_{i(1)}$ containing $p$. Let $W(t)$ be Brownian motion in $\mathbf{C}$, and let $\sigma_{1}$ be the first time that $p+W(t)$ hits the boundary of $c_{i(1)}\left(m_{i(1)}\right)$. For $0 \leqslant t \leqslant \sigma_{1}$, let $B(t)=c_{i(1)}^{-1}(p+W(t))$. Next, let $m_{i(2)}$ be a patch containing $B\left(\sigma_{1}\right)$, and let $\sigma_{2}$ be the first time that $c_{i(2)}\left(B\left(\sigma_{1}\right)\right)+W(t)-$ $W\left(\sigma_{1}\right)$ hits the boundary of $m_{i(2)}$. For $\sigma_{1} \leqslant t \leqslant \sigma_{2}$, let

$$
B(t)=c_{i(2)}^{-1}\left(c_{i(2)}\left(B\left(\sigma_{1}\right)\right)+W(t)-W\left(\sigma_{1}\right)\right),
$$

and note that we have defined $B(t)$ to be continuous at $\sigma_{1}$. For $k>2$, define $\sigma_{k}$ and $B(t), \sigma_{k} \leqslant t \leqslant \sigma_{k+1}$, analogously.

We also need

Lemma 9. Let $p(N)>0$. Then the fundamental group of $N$ is the free group with $n(N)-1$ generators. The generators may be taken to be the cycles around single points (except one) and the canonical generators of the tori.

Suppose $F$ is not constant. Let $G(M)$ be the fundamental group of $M$ with base point $p$, and let $G(N)$ be the fundamental group of $N$ with base point $F(p)$. Let $\left\{\alpha_{i}^{M}\right\},\left\{\alpha_{i}^{N}\right\}$ be the generators of $G(M), G(N)$, resp., as described in Lemma 9. Choose 2 generators $\alpha_{1}^{M}, \alpha_{2}^{M}$ of $G(M)$ which are generators of a torus, and let $\alpha_{3}^{M}, \ldots, \alpha_{n(M)-1}^{M}$ be the remaining generators. Let $H(M)$ be the smallest normal subgroup of $G(M)$ containing $\alpha_{3}^{M}, \ldots, \alpha_{n(M)-1}^{M}$ and the commutator $[G(M), G(M)]$. Let $\hat{G}(M)=G(M) / H(M)$, and note that $\hat{G}(M)$ is isomorphic to $\mathbf{Z} \times \mathbf{Z}$. 
Let $\mathrm{O}, \mathrm{O}_{2}, \mathrm{O}_{3}$ be neighborhoods of $p$, contained in $m_{i(1)}$, whose images under $c_{i(1)}$ are discs of radii $\epsilon, 2 \epsilon, 3 \epsilon$, respectively. Let $B^{Q}(t)$ be the Brownian motion on $M$ whose initial distribution $B(0)$ is the measure $Q$, concentrated on the boundary of 0 . Let $\tau_{0}=0$; given $\tau_{i}$, let $\tau_{i+1}$ be the first time after $\tau_{i}$ for which $B\left(\tau_{i+1}\right) \in \partial O$, and $\left\{B(t): \tau_{i} \leqslant t \leqslant \tau_{i+1}\right\}$ corresponds to a nontrivial element of $\hat{G}(M)$. Note that since $M$ is compact except for deleted points, all $\tau_{i}$ are finite.

Next, let $x_{n}=B^{Q}\left(\tau_{n}\right)$, and note that $x_{n}$ is a Markov process with respect to the fields $\sigma\left\{B(t): t \leqslant \tau_{n}\right\}$. Let $m$ be the measure on $\partial O$ induced by Lebesgue measure on the circle $c_{i(1)}(\partial 0)$. By a theorem of Harris [6], it can be shown that $x_{n}$ has an invariant measure. We set $Q$ equal to this measure.

Let $\hat{X}_{i}$ be the element of $\hat{G}(M)$ corresponding to $\left\{B^{Q}(t): 0 \leqslant t \leqslant \tau_{i}\right\}$. Since $\hat{G}(M) \cong \mathbf{Z} \times \mathbf{Z}$, we may regard $\hat{X}_{i}$ as a random walk on $\mathbf{R}^{2}$. Let $\Delta_{i}=$ $\hat{X}_{i}-\hat{X}_{i-1}$. Then, $\left\{\Delta_{i}\right\}$ is a stationary process with $E\left|\Delta_{i}\right|^{4}<\infty$. Moreover the process is symmetric, so $\Delta_{i}$ and $-\Delta_{i}$ have the same distribution. Using the central limit theorem (see Theorem 9 of Phillip [9]), it is shown that $\hat{X}_{i}$ is recurrent. By Lemma 3.1 of [1], it follows that $X_{i}$ is recurrent.

Now, $F$ induces a map $F_{H}$ from $H /[H, H]$ to $G(N) /[G(N), G(N)]$. Since these are commutative groups with difference in dimension at least 3 , it follows that there must be at least 3 generators $\alpha_{1}^{N}, \alpha_{2}^{N}, \alpha_{3}^{N}$ of $G(N)$ which generate a subgroup of $G(N) /[G(N), G(N)]$ modulo $F_{H}(H /[H, H])$ isomorphic to $\mathbf{Z} \times \mathbf{Z} \times \mathbf{Z}$. Let $H(N)$ be the smallest normal subgroup of $G(N)$ containing $\alpha_{4}^{N}, \ldots, \alpha_{n(N)-1}^{N}$ and $[G(N), G(N)]$, and let $\hat{G}(N)=G(N) / H(N)$. Then, $F$ induces a map $\hat{F}$ from $\hat{G}(M)$ to $\hat{G}(N)$.

Using Brownian motion on $N$, we construct as before an invariant measure $Q$ on $F(O)$ and a random walk $\hat{Y}_{i}$ from $F(B(t))$. By a theorem of Lévy [1], $F(B(t))$ is Brownian motion on $N$ with a new time scale. By the Borel-Cantelli lemma, $\hat{Y}_{i}$ is transient. Davis's argument [1] then shows that the random walk $Y_{i}$ induced by $F(B(t))$ with $B(0)=p$ is also transient. But then $\hat{F}\left(X_{i}\right)=Y_{i}$, and $X_{i}$ is recurrent. This contradiction shows that $F$ must be constant.

\section{REFERENCES}

1. B. Davis, Picard's theorem and Brownian motion, Trans. Amer. Math. Soc. 213 (1975), 353-362.

2. J. Eells and L. Lemaire, $A$ report on harmonic maps, Bull. London Math. Soc. 10 (1978), 1-68.

3. R. E. Greene and $\mathrm{H}$. Wu, Function theory on manifolds which possess a pole, Lecture Notes in Math., vol. 699, Springer-Verlag, Berlin, 1979.

4. S. I. Goldberg, T. Ishihara and N. C. Petridis, Mappings of bounded dilatation of Riemannian manifolds, J. Differential Geom. 10 (1975), 619-630.

5. S. I. Goldberg and T. Ishihara, Harmonic quasiconformal mappings of Riemannian manifolds, Amer. J. Math. 98 (1976), 225-240.

6. T. E. Harris, The existence of stationary measures for certain Markov processes, Proc. of the 3rd Berkeley Symp. on Math. Stat. and Prob., Vol. II (1956), 113-124. 
7. W. S. Kendall, Brownian motion and a generalised little Picard's theorem, Trans. Amer. Math. Soc. (to appear).

8. H. P. McKean, Jr., Stochastic integrals, Academic Press, New York and London, 1969.

9. W. Phillip, The central limit theorem for mixing sequences of random variables, Z. Wahrsch. Verv. Geb. 12 (1969), $155-171$.

10. M. Pinsky, Stochastic Riemannian geometry, Probabilistic Analysis and Related Topics, Vol. I (A. T. Barucha-Reid, ed.), Academic Press, New York, 1978.

11. J.-J. Prat, Etude asymptotique et convergence angulaire du mouvement brownien sur une variété d̀ courbure negative, C. R. Acad. Sci. Paris, Ser. A 280 (1975), 1539-1542.

12. D. W. Strook and S. R. S. Varadhan, On the support of diffusion processes with applications to the strong maximum principle, Proc. 6th Berkeley Symp. on Math. Stat. and Prob. III (1970), 333-360.

DEPARTMENT OF MATHEMATICS, UNIVERSITY OF ILLINOIS, URBANA, ILLINOIS 61801

DEPARTMENT OF MATHEMATICS, UNIVERSITY OF TEXAS AT AUSTIN, AUSTIN, TEXAS 78712 